\title{
3D DOMAIN DECOMPOSITION METHOD COUPLING CONFORMING AND NONCONFORMING FINITE ELEMENTS
}

\author{
Abdellatif Agouzal ${ }^{1}$, Laurence Lamoulie ${ }^{2}$ and Jean-Marie Thomas $^{3}$
}

\begin{abstract}
This paper deals with the solution of problems involving partial differential equations in $\mathbb{R}^{3}$. For three dimensional case, methods are useful if they require neither domain boundary regularity nor regularity for the exact solution of the problem. A new domain decomposition method is therefore presented which uses low degree finite elements. The numerical approximation of the solution is easy, and optimal error bounds are obtained according to suitable norms.
\end{abstract}

AMS Subject Classification. 65N30.

Received: November 28, 1995. Revised: October 2, 1998.

\section{INTRODUCTION}

In three dimensions, the domain boundary regularity is obviously root of difficulties. For example some bounded polyhedral domain have a continuous but not Lipschitzian boundary (cf. Fig. 1).

In this example, near the contact points between the two bars, nor Lipschitzian function exists that can fit the domain boundary.

Numerical solution is harder because of the lack of regularity. Domain decomposition sometimes allows to overcome this problem: a clever decomposition may lead to regular bounded subdomains, from an initial regular domain. For Figure 1, a good choice is obviously the following one (cf. Fig. 2).

Matching unknowns on the interface is one of the difficulties one is faced with in domain decomposition methods. Mesh parameters must not imposed on both sides of the interface by the chosen matching conditions. Moreover numerical solution must be easy on sequential or parallel computers.

In the context of non matching grids, to our knowledge, three approaches are considered in literature: mortar element methods, in a primal formulation [6] or in a dual formulation [2], hybrid methods [3,13], and primal-dual coupling methods $[4,10]$.

Allowing to achieve this goal, a domain decomposition method using low degree finite elements is presented here. For the sake of shortness and simplicity, the method is exposed for the following model problem.

Assuming that $f$ is a given function in $L^{2}(\Omega)$, find $u$ solution of

$$
\begin{cases}-\Delta u=f & \text { in } \Omega, \\ u=0 & \text { on } \partial \Omega .\end{cases}
$$

Keywords and phrases. Domain decomposition, hybrid finite elements methods.

${ }^{1}$ Université Claude Bernard - Lyon I and UMR 5585, 43 bd. du 11 Novembre 1918, 69622 Villeurbanne, France.

e-mail: agouzal@iris.univ-lyon1.fr

2 Ecole d'Ingénieurs en Génie des Systèmes Industriels, 17041 La Rochelle, France.

${ }^{3}$ Laboratoire de Mathématiques Appliquées pour l'Industrie, UPRES A 5033, Université de Pau, 64000 Pau, France.

(C) EDP Sciences, SMAI 1999 


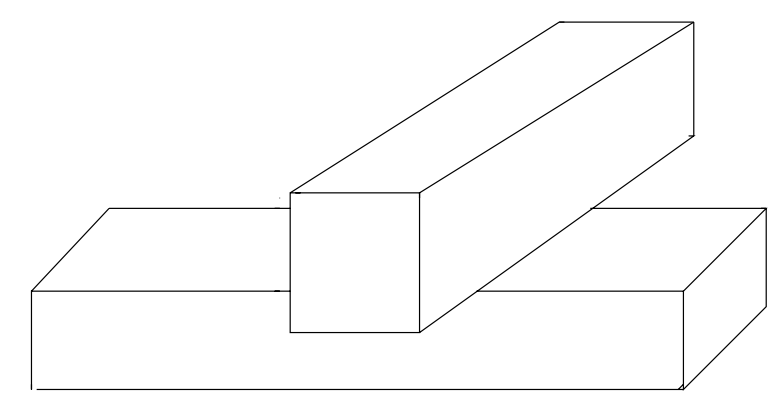

Figure 1. A non Lipschitzian domain
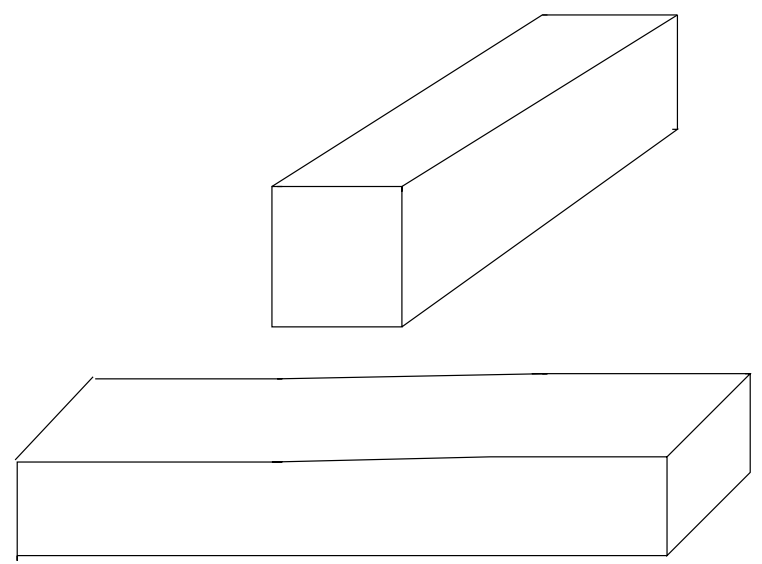

FiguRE 2. Domain decomposition with regular bounded subdomains.

Interpolation results are firstly proved, which allows to analyse a domain decomposition method coupling conforming and nonconforming finite elements. A new formulation is then established that couples primal and dual variables. The idea was first introduce in $[4,10]$, and used for example in [5] for the coupling of Stokes equations. Using low degree spaces for numerical approximation, optimal error bounds are obtained without any condition on meshes and restricting regularity requirements on the solution.

\section{A THREE DIMENSIONAL INTERPOLATION OPERATOR}

Let $T$ be a tetrahedron, let us denote its faces by $F_{i}, i=1, \ldots, 4$. It is well known and immediate to prove that for each $v \in H^{1}(T)$, there exists a unique affine function $\Pi_{h} v \in P_{1}(T)$ such that:

$$
\forall i=1, \ldots, 4 \quad \int_{F_{i}} \Pi_{T} v \mathrm{~d} \sigma=\int_{F_{i}} v \mathrm{~d} \sigma
$$

So $\Pi_{T}$ define an interpolation operator; associated interpolation error bounds are now to be determined. The following result will be helpful:

Lemma 2.1. As operator from $H^{1}(T)$ onto $P_{1}(T), \Pi_{T}$ is continuous and for all $u \in H^{1}(T)$ the following inequality holds:

$$
\left|\Pi_{T} u\right|_{1, T} \leq|u|_{1, T} .
$$


Proof. Assuming that $u \in H^{1}(T)$, by Green formula we have for all regular test function $v$ :

$$
\left(u-\Pi_{T} u, v\right)_{1, T}=-\int_{T}\left(u-\Pi_{T} u\right) \Delta v \mathrm{~d} x+\sum_{i=1}^{4} \int_{F_{i}}\left\{\frac{\partial v}{\partial n_{i}}\left(u-\Pi_{T} u\right) \mathrm{d} \sigma\right\}
$$

Then for all affine function $v$, sine $\Delta v=0$ and $\int_{F_{i}}\left(u-\Pi_{T} u\right) \mathrm{d} \sigma=0, i=1, \ldots, 4$, this implies that

$$
\left(u-\Pi_{T} u, v\right)_{1, T}=0 .
$$

So $\Pi_{T} u$ is he orthogonal projection of $u$ onto $P_{1}(T)$ for the scalar product of $H^{1}(T)$ and so

$$
\left|\Pi_{T} u\right|_{1, T} \leq|u|_{1, T} .
$$

Using Lemma 2.1, standard arguments of finite elements methods theory and functional interpolation of Hilbert spaces, the following interpolation error bounds holds (see also [1] for similar result in two-dimensional case).

Theorem 2.1. There exists a constant $C$ such that, for all $u \in H^{1+\sigma}(T), 0 \leq \sigma \leq 1$, we have

$$
\left|u-\Pi_{T} u\right|_{1, T} \leq C h^{\sigma}|u|_{1+\sigma, T} .
$$

\section{CONFORMING-NONCONFORMING HYBRID FORMULATION}

The following model problem is studied:

$\mathcal{P}$ : Assuming that $f$ is a given function in $L^{2}(\Omega)$, find $u$ solution of

$$
\begin{cases}-\Delta u=f & \text { in } \Omega, \\ u=0 & \text { on } \partial \Omega .\end{cases}
$$

Assume that $\Omega$ is an open connected subset of $\mathbb{R}^{3}$, which boundary $\partial \Omega$ is regular enough but may be not Lipschitzian. $\Omega$ is divided into two non overlapping subdomains $\Omega_{1}$ and $\Omega_{2}$ supposed connected for simplicity. The interface between $\Omega_{1}$ and $\Omega_{2}$ is called $\Sigma$, and $\Gamma_{i}=\partial \Omega_{i} / \Sigma, i=1,2$. For sake of simplicity, it is supposed that $\Gamma_{i} \neq \emptyset, i=1,2$.

Let $u_{i}, i=1,2$ denote the restriction of $u$ to the subdomain called $\Omega_{i}, i=1,2$.

\subsection{Continuous formulation}

Few notations are needed:

$$
\begin{aligned}
V_{i} & =H_{0, \Gamma_{i}}^{1}\left(\Omega_{i}\right)=\left\{v \in H^{1}\left(\Omega_{i}\right) \text { such that } v_{\mid \Gamma_{i}}=0\right\}, \quad i=1,2, \\
H_{0,0}^{1 / 2}(\Sigma) & =\left\{v_{\mid \Sigma}, v \in V_{1}\right\}=\left\{v_{\mid \Sigma}, v \in V_{2}\right\} \\
\Lambda & =H^{-1 / 2}(\Sigma) \quad \text { dual space of } H_{0,0}^{1 / 2}(\Sigma) .
\end{aligned}
$$

A continuous formulation of $\mathcal{P}$ is established dualizing the traces matching equation on the interface $\Sigma$.

\section{Continuous hybrid formulation:}

$\mathcal{P}_{H}$ : Find $\left(u_{1}, u_{2}, \lambda\right) \in V_{1} \times V_{2} \times \Lambda$ such that

$$
\left\{\begin{array}{l}
\forall\left(v_{1}, v_{2}\right) \in V_{1} \times V_{2}, \sum_{i=1}^{2} \int_{\Omega_{i}} \operatorname{grad} u_{i} \cdot \operatorname{grad} v_{i} \mathrm{~d} x-\left\langle\lambda,\left(v_{1}-v_{2}\right)_{\mid \Sigma}\right\rangle=\sum_{i=1}^{2} \int_{\Omega_{i}} f v_{i} \mathrm{~d} x \\
\forall \mu \in \Lambda,\left\langle\mu,\left(u_{1}-u_{2}\right)_{\mid \Sigma}\right\rangle=0 .
\end{array}\right.
$$

where $\langle.,$.$\rangle denotes the duality pairing between H^{-1 / 2}(\Sigma)$ and $H_{0,0}^{1 / 2}(\Sigma)$. 
As it was shown by [12], the following result holds:

Theorem 3.1. The problem $\left(\mathcal{P}_{H}\right)$ has a unique solution satisfying the following equalities:

$$
\left\{\begin{array}{l}
u_{i}=u_{\mid \Omega_{i}}, \quad i=1,2 \\
\lambda=\frac{\partial u}{\partial n_{1}} \text { in } H^{-1 / 2}(\Sigma) .
\end{array}\right.
$$

Remark 1. The solution would be also unique if a Dirichlet condition were imposed only on a subset of non zero measure of $\Gamma_{1}$ or $\Gamma_{2}$. The local problems are well-posed if meas $\left(\Gamma_{i}\right) \neq 0, i=1,2$.

Remark 2. The variational problem $\left(\mathcal{P}_{H}\right)$ is equivalent to the saddle point problem for the Lagrangian

$$
\mathcal{L}\left(v_{1}, v_{2}, \mu\right)=\sum_{i=1}^{2}\left|v_{i}\right|_{1, \Omega_{i}}^{2} \mathrm{~d} x-2 \sum_{i=1}^{2} \int_{\Omega_{i}} f v_{i} \mathrm{~d} x-2\left\langle\mu,\left(v_{1}-v_{2}\right)_{\mid \Sigma}\right\rangle .
$$

\subsection{Discrete formulation}

Assume that $\Omega, \Omega_{1}$ and $\Omega_{2}$ are now polyhedral open sets, without any crack. For $i=1,2$, let $\mathcal{T}_{i}$ denote a regular triangulation according to Ciarlet [8] of $\Omega_{i}$, which mesh size is called $h_{i}$. Neither compatibility condition between triangulations, nor between mesh sizes $h_{1}$ and $h_{2}$ are imposed on both sides of $\Sigma$.

The discretization will need the following spaces and subsets:

$$
V_{1, h}=\left\{v_{h} \in V_{1} ; \forall T \in \mathcal{T}_{1, h}, v_{\mid T} \in P_{1}(T)\right\} .
$$

Let $S_{h}$ denote the set of tetrahedral faces included in $\Omega_{2}$ i.e.:

$$
S_{h}=\left\{F=\partial T_{1} \cap \partial T_{2},\left(T_{1}, T_{2}\right) \in\left(\mathcal{T}_{2, h}\right)^{2} \text { and meas }\left(\partial T_{1} \cap \partial T_{2}\right) \neq 0\right\}
$$

and $S_{h}^{\Gamma}\left(S_{h}^{\Sigma}\right)$ denote the set of tetrahedral faces included in $\Gamma_{2}($ in $\Sigma)$ i.e.:

$$
\begin{aligned}
& S_{h}^{\Gamma}=\left\{F=\partial T \cap \Gamma_{2}, T \in \mathcal{T}_{2, h} \text { and } \operatorname{meas}\left(\partial T \cap \Gamma_{2}\right) \neq 0\right\} ; \\
& S_{h}^{\Sigma}=\left\{F=\partial T \cap \Sigma, T \in \mathcal{T}_{2, h} \text { and } \operatorname{meas}(\partial T \cap \Sigma) \neq 0\right\} .
\end{aligned}
$$

The spaces $V_{2, h}$ and $\Lambda_{h}$ are defined by:

$$
\begin{aligned}
V_{2, h} & =\left\{v_{h} \in L^{2}\left(\Omega_{2}\right) ; \forall T \in \mathcal{T}_{2, h}, v_{h \mid T} \in P_{1}(T) ;\right. \\
\forall F & \left.=\partial T_{1} \cap \partial T_{2} \in S_{h}, \int_{F} v_{h \mid T_{1}} \mathrm{~d} \sigma=\int_{F} v_{h \mid T_{2}} \mathrm{~d} \sigma, \forall F \in S_{h}^{\Gamma}, \int_{F} v_{h} \mathrm{~d} \sigma=0\right\}
\end{aligned}
$$

and

$$
\Lambda_{h}=\left\{\mu_{h} \in L^{2}(\Sigma) ; \forall F \in S_{h}^{\Sigma}, \mu_{h \mid F} \in P_{0}(F)\right\}
$$

As $V_{2, h}$ is of nonconforming space type, it must equipped with the following broken norm

$$
\forall v_{2, h} \in V_{2, h}, \quad\left|v_{2, h}\right|_{1, h}=\left(\sum_{T \in \mathcal{T}_{2, h}}\left|v_{2, h}\right|_{1, T}^{2}\right)^{\frac{1}{2}}
$$


The following discrete formulation is obtained from the $\left(\mathcal{P}_{H}\right)$ formulation:

\section{Discrete Hybrid conforming-nonconforming formulation:}

$\mathcal{P}_{H D}$ : Find $\left(u_{1, h}, u_{2, h}, \lambda_{h}\right) \in V_{1, h} \times V_{2, h} \times \Lambda_{h}$ such that

$$
\left\{\begin{array}{l}
\forall\left(v_{1, h}, v_{2, h}\right) \in V_{1, h} \times V_{2, h}, \\
\int_{\Omega_{1}} \operatorname{grad} u_{1, h} \cdot \operatorname{grad} v_{1, h} \mathrm{~d} x+\sum_{T \in \mathcal{T}_{2, h}} \int_{T} \operatorname{grad} u_{2, h} \operatorname{grad} v_{2, h} \mathrm{~d} x-\int_{\Sigma} \lambda_{h}\left(v_{1, h}-v_{2, h}\right) \mathrm{d} \sigma= \\
\forall \mu_{h} \in \Lambda_{h}, \quad \int_{\Sigma} \mu_{h}\left(u_{1, h}-u_{2, h}\right) \mathrm{d} \sigma=0
\end{array}\right.
$$

where $\bar{f}$ denotes the constant by triangle function, that is $L^{2}\left(\Omega_{2}\right)$-projection of $f$ on the piecewise constant finite element space. Modifying $f$ in the right-hand side of the equation on $\Omega_{2}$ is equivalent to evaluate $\int_{\Omega_{2}} f v_{2, h}$ using numerical integration formula.

The following result will be helpful:

Lemma 3.1. There exists a constant $C>0$, independent of mesh parameters such that, if $\bar{b}(.,$.$) is the bilinear$ form defined on $V_{2} \times \Lambda_{h}$ by

$$
\bar{b}\left(v_{2}, \mu_{h}\right)=\int_{\Sigma} \mu_{h} v_{2} \mathrm{~d} \sigma
$$

then

$$
\sup _{v_{2} \in V_{2}} \frac{\bar{b}\left(v_{2}, \mu_{h}\right)}{\left\|v_{2}\right\|_{1, \Omega_{2}}} \geq C\left\|\mu_{h}\right\|_{-1 / 2, \Sigma}, \quad \text { for all } \mu_{h} \in \Lambda_{h}
$$

Proof. Introducing $v_{2}$ solution to:

$$
\begin{cases}-\Delta v_{2}=0 & \text { in } \Omega_{2} \\ \partial v_{2} / \partial n=\mu_{h} & \text { on } \Sigma \\ v_{2}=0 & \text { on } \Gamma_{2}\end{cases}
$$

the Green formula leads to

$$
\bar{b}\left(v_{2}, \mu_{h}\right)=\left|v_{2}\right|_{1, \Omega_{2}}^{2} .
$$

Moreover trace theorem provides

$$
\left\|\mu_{h}\right\|_{-1 / 2, \Sigma} \leq\left\|v_{2}\right\|_{1, \Omega_{2}} .
$$

Equivalence of norm and semi-norm in $H_{0, \Gamma_{2}}^{1}\left(\Omega_{2}\right)$ allows to conclude.

The following theorem can then be proved.

Theorem 3.2. Problem $\left(\mathcal{P}_{H D}\right)$ has a unique solution.

Proof. In the formulation of $\left(P_{H D}\right)$ appears the bilinear form $a(.,$.$) :$

$$
a\left(\left(u_{1, h}, u_{2, h}\right),\left(v_{1, h}, v_{2, h}\right)\right)=\int_{\Omega_{1}} \operatorname{grad} u_{1, h} \cdot \operatorname{grad} v_{1, h} \mathrm{~d} x+\sum_{T \in \mathcal{T}_{2, h}} \int_{T} \operatorname{grad} u_{2, h} \operatorname{grad} v_{2, h} \mathrm{~d} x .
$$

Therefore for each $\left(v_{1, h}, v_{2, h}\right)$ :

$$
a\left(\left(v_{1, h}, v_{2, h}\right),\left(v_{1, h}, v_{2, h}\right)\right)=\int_{\Omega_{1}}\left\|\operatorname{grad} v_{1, h}\right\|^{2}+\sum_{T \in \mathcal{T}_{2, h}} \int_{T}\left\|\operatorname{grad} v_{2, h}\right\|^{2} \mathrm{~d} x
$$


$\left(P_{H D}\right)$ solution $\left(u_{1, h}, u_{2, h}\right)$ belongs to the so-called $V_{h}$ product space defined by

$$
V_{h}=V_{1, h} \times V_{2, h}
$$

The bilinear form $a(.,$.$) is continuous and elliptic with respect to V_{h}$ norm.

If $a\left(\left(u_{1, h}, u_{2, h}\right),\left(u_{1, h}, u_{2, h}\right)\right)=0$, then $\left|u_{1, h}\right|_{1, \Omega_{1}}=0$ and, for all $T \in \mathcal{T}_{2, h}, \quad\left|u_{2, h}\right|_{1, T}=0$, so $u_{1, h}=0$ and $u_{2, h}=0$.

We shall now prove that the bilinear form $b(.,$.$) defined by$

$$
b\left(\left(v_{1, h}, v_{2, h}\right), \mu_{h}\right)=\int_{\Sigma} \mu_{h}\left(v_{1, h}-v_{2, h}\right) \mathrm{d} \sigma
$$

must satisfy the following inf-sup condition: there exist a positive constant $C$, independent of $h$, such that:

$$
\inf _{\mu_{h} \in \Lambda_{h}, \mu_{h} \neq 0} \sup _{\left(v_{1, h}, v_{2, h}\right) \in V_{h}} \frac{b\left(\left(v_{1, h}, v_{2, h}\right), \mu_{h}\right)}{\left\|\mu_{h}\right\|_{-1 / 2, \Sigma}\left\|\left(v_{1, h}, v_{2, h}\right)\right\|_{V_{h}}} \geq C .
$$

One can notice that this condition is satisfied as soon as we have

$$
\inf _{\mu_{h} \in \Lambda_{h}, \mu_{h} \neq 0} \sup _{\left(0, v_{2, h}\right) \in V_{h}} \frac{b\left(\left(0, v_{2, h}\right), \mu_{h}\right)}{\left\|\mu_{h}\right\|_{-1 / 2, \Sigma}\left\|v_{2, h}\right\|_{V_{2, h}}} \geq C
$$

that is to say

$$
\inf _{\mu_{h} \in \Lambda_{h}, \mu_{h} \neq 0} \sup _{v_{2, h} \in V_{2, h}} \frac{\bar{b}\left(v_{2, h}, \mu_{h}\right)}{\left\|\mu_{h}\right\|_{-1 / 2, \Sigma}\left\|v_{2, h}\right\|_{V_{2, h}}} \geq C .
$$

Fortin's lemma is used to demonstrate this result [7]. Indeed

$$
\inf _{\mu_{h} \in \Lambda_{h}, \mu_{h} \neq 0} \sup _{v_{2, h} \in V_{2, h}} \frac{\bar{b}\left(v_{2, h}, \mu_{h}\right)}{\left\|\mu_{h}\right\|_{-1 / 2, \Sigma}\left\|v_{2, h}\right\|_{V_{2, h}}} \geq \inf _{\mu_{h} \in \Lambda_{h}, \mu_{h} \neq 0} \sup _{v_{2} \in V_{2}} \frac{\bar{b}\left(\Pi_{h} v_{2}, \mu_{h}\right)}{\left\|\mu_{h}\right\|_{-1 / 2, \Sigma}\left\|\Pi_{h} v_{2}\right\|_{V_{2, h}}}
$$

so

$$
\inf _{\mu_{h} \in \Lambda_{h}, \mu_{h} \neq 0} \sup _{v_{2, h} \in V_{2, h}} \frac{\bar{b}\left(v_{2, h}, \mu_{h}\right)}{\left\|\mu_{h}\right\|_{-1 / 2, \Sigma}\left\|v_{2, h}\right\|_{V_{2, h}}} \geq \inf _{\mu_{h} \in \Lambda_{h}, \mu_{h} \neq 0} \sup _{v_{2} \in V_{2}} \frac{\bar{b}\left(v_{2}, \mu_{h}\right)}{\left\|\mu_{h}\right\|_{-1 / 2, \Sigma}\left|v_{2}\right|_{1, \Omega_{2}}}
$$

because the interpolation operator is continuous. Lemma 2.1 is then providing the result.

Existence and uniqueness of the solution are established.

Theorem 3.3. If the solution to $P_{H}$ is such that $u_{i}=u_{\mid \Omega_{i}} \in H^{\sigma_{i}}\left(\Omega_{i}\right)$ with $1<\sigma_{i} \leq 2$, $i=1,2$, then we have the following error bound for discrete problem $P_{H D}$ :

$$
\left|u_{1}-u_{1, h}\right|_{1, \Omega_{1}}+\left(\sum_{T \in \mathcal{T}_{2, h}}\left|u_{2}-u_{2, h}\right|_{1, T}^{2}\right)^{1 / 2} \leq C\left(h_{1}^{\sigma_{1}-1}\left\|u_{1}\right\|_{\sigma_{1}, \Omega_{1}}+h_{2}^{\sigma_{2}-1}\left\|u_{2}\right\|_{\sigma_{2}, \Omega_{2}}\right) .
$$

Proof. A new formulation is necessary to make the proof easier: the weak matching condition is relaxed using a primal hybrid formulation [7,12]. Arguments introduced by [12] allow to conclude the proof. In order to get rid of regularity conditions on exact solution, introducing interpolation operators is useful, namely:

- for nonconforming variable, $\Pi_{h}$ operator, for which error interpolation bounds have been given in Theorem 1 ,

- for conforming variable, to avoid supposing that $u \in H^{3 / 2+\epsilon}\left(\Omega_{1}\right)$, one can use a Clément interpolation operator [9], which is defined from $V_{1}$ onto $V_{1, h}$. 


\section{Equivalence With LOW DEGREe SEMi-PRIMAL SEMI-DUAL FORMUlation FOR 3D}

Our aim is to establish a semi-primal semi-dual formulation $[4,10]$, that is a formulation using for example the primal unknown $u$ on $\Omega_{1}$ and the dual unknown $\mathbf{p}$ on $\Omega_{2}$.

The discrete formation comes from the conforming-nonconforming hybrid formulation $\left(P_{H D}\right)$, previously established. Indeed, if one chooses:

- $u_{1, h}$ used in the conforming-nonconforming hybrid formulation

- $\mathbf{p}_{2, h}$ defined on $\Omega_{2}$ by:

$$
\mathbf{p}_{2, h}=\operatorname{grad} u_{2, h}-\frac{\bar{f}}{3}\left(\mathbf{x}-\mathbf{x}_{T}\right), \quad \text { for all } T \in \mathcal{T}_{2, h}
$$

where $u_{2, h}$ is the nonconforming unknown involed in the conforming-nonconforming hybrid formulation; $\mathbf{x}_{T}$ the triangle barycenter and $\bar{f}$ the mean value of $f$ on $T$. Then $\left(u_{1, h}, \mathbf{p}_{2, h}\right)$ is solution of semi-primal semi-dual formulation associated to problem $P$. The above construction of $\mathbf{p}_{2, h}$ from $u_{2, h}$ is similar in 3D to the one given in $2 \mathrm{D}$ by [11].

Let $W_{2, h}$ defined by

$$
W_{2, h}=\left\{\mathbf{p}_{h} \in H\left(\operatorname{div}, \Omega_{2}\right), \forall T \in \mathcal{T}_{2, h}, \mathbf{p}_{h \mid T} \in R T_{1}(T)\right\}
$$

where $R T_{1}(T)=\left(P_{0}(T)\right)^{3}+\mathbf{x} P_{0}(T)$.

Some preliminary results are to be shown, what is done in the following lemmas.

Lemma 4.1. The vectorial function $\mathbf{p}_{2, h}$ defined by (4.1) belongs to $H\left(\operatorname{div}, \Omega_{2}\right)$.

Proof. On each $T \in \mathcal{T}_{2, h}, \mathbf{p}_{2, h} \in\left(L^{2}(T)\right)^{2}$ and $-\operatorname{div} \mathbf{p}_{2, h}=\bar{f}$. One just needs then to show that

$$
\forall T_{1}, T_{2} \in \mathcal{T}_{2, h} \text {, with meas }\left(\partial T_{1} \cap \partial T_{2}\right) \neq 0, \mathbf{p}_{2, h} . n_{1}+\mathbf{p}_{2, h} . n_{2}=0 \text { on } F=\partial T_{1} \cap \partial T_{2} .
$$

Let $\left[\mathbf{p}_{2, h} . n\right]$ denote the normal jump through any face $F$ defined by

$$
\left[\mathbf{p}_{2, h} . n\right]=\mathbf{p}_{2, h} . n_{1}+\mathbf{p}_{2, h} \cdot n_{2} .
$$

To obtain $\left[\mathbf{p}_{2, h} . n\right]=0$, one can show that $\int_{F}\left[\mathbf{p}_{2, h} . n\right] v_{h} \mathrm{~d} \sigma=0$, for the basis function $v_{h} \in V_{2, h}$ associated to $F$ defined by

$$
\int_{F} v_{h} \mathrm{~d} \sigma=1
$$

and

$$
\text { for all face } F^{\prime} \neq F, \int_{F^{\prime}} v_{h} \mathrm{~d} \sigma=0 .
$$

With the definition of $\left[\mathbf{p}_{2, h} . n\right]$ and Green formulae, we have

$$
\int_{F}\left[\mathbf{p}_{2, h} . n\right] v_{h} \mathrm{~d} \sigma=\int_{T_{1}} \mathbf{p}_{2, h} \operatorname{grad} v_{h} \mathrm{~d} x+\int_{T_{2}} \mathbf{p}_{2, h} \operatorname{grad} v_{h} \mathrm{~d} x+\int_{T_{1}} \operatorname{div} \mathbf{p}_{2, h} v_{h} \mathrm{~d} x+\int_{T_{2}} \operatorname{div} \mathbf{p}_{2, h} v_{h} \mathrm{~d} x
$$

and from $\mathbf{p}_{2, h}$ definition, we obtain

$$
\begin{aligned}
\int_{F}\left[\mathbf{p}_{2, h} . n\right] v_{h} \mathrm{~d} \sigma= & \int_{T_{1}} \operatorname{grad} u_{2, h} \operatorname{grad} v_{h} \mathrm{~d} x+\int_{T_{2}} \operatorname{grad} u_{2, h} \operatorname{grad} v_{h} \mathrm{~d} x \\
& -\frac{\bar{f}}{3} \int_{T_{1}}\left(\mathbf{x}-\mathbf{x}_{T_{1}}\right) \operatorname{grad} v_{h} \mathrm{~d} x-\frac{\bar{f}}{3} \int_{T_{2}}\left(\mathbf{x}-\mathbf{x}_{T_{1}}\right) \operatorname{grad} v_{h} \mathrm{~d} x-\int_{T_{1}} \bar{f} v_{h} \mathrm{~d} x-\int_{T_{2}} \bar{f} v_{h} \mathrm{~d} x .
\end{aligned}
$$


Using the facts that $u_{2, h}$, being the solution with right hand side $\bar{f}$, verifies:

$$
\int_{T_{1}} \operatorname{grad} u_{2, h} \operatorname{grad} v_{h} \mathrm{~d} x+\int_{T_{2}} \operatorname{grad} u_{2, h} \operatorname{grad} v_{h} \mathrm{~d} x=\int_{T_{1}} \bar{f} v_{h} \mathrm{~d} x+\int_{T_{2}} \bar{f} v_{h} \mathrm{~d} x
$$

and that $\forall T \in \mathcal{T}_{2, h}, \int_{T}\left(\mathbf{x}-\mathbf{x}_{T}\right) \mathrm{d} x=0$, we get the result

$$
\int_{F}\left[\mathbf{p}_{2, h} . n\right] v_{h} \mathrm{~d} \sigma=0
$$

Lemma 4.2. The vectorial function $\mathbf{p}_{2, h}$ belongs to $W_{2, h}$, where the space $W_{2, h}$ is defined in (3.2).

Lemma 4.3. The function $\mathbf{p}_{2, h}$ satisfies:

$$
\forall \mathbf{q}_{2, h} \in W_{2, h}, \text { with } \operatorname{div} \mathbf{q}_{2, h}=0, \quad \int_{\Omega_{2}} \mathbf{p}_{2, h} \mathbf{q}_{2, h} \mathrm{~d} x=\int_{\Sigma} u_{1, h} \mathbf{q}_{2, h} . n \mathrm{~d} \sigma .
$$

Proof. Using Lemma 4.4 and 4.5, we have $\mathbf{p}_{2, h} \in W_{2, h}$

$$
\begin{aligned}
\int_{\Omega_{2}} \mathbf{p}_{2, h} \mathbf{q}_{2, h} \mathrm{~d} x & =\sum_{T \in \mathcal{T}_{2, h}} \int_{T}\left(\operatorname{grad} u_{2, h}-\frac{\bar{f}}{3}\left(\mathbf{x}-\mathbf{x}_{T}\right)\right) \mathbf{q}_{2, h} \mathrm{~d} x \\
& =\sum_{T \in \mathcal{T}_{2, h}} \int_{T} \operatorname{grad} u_{2, h} \mathbf{q}_{2, h} \mathrm{~d} x-\sum_{T \in \mathcal{T}_{2, h}} \int_{T} \frac{\bar{f}}{3}\left(\mathbf{x}-\mathbf{x}_{T}\right) \mathbf{q}_{2, h} \mathrm{~d} x .
\end{aligned}
$$

Because $\mathbf{q}_{2, h} \in W_{2, h}, \mathbf{q}_{2, h}$ can be written as $\mathbf{q}_{2, h}=\underline{\alpha}+\beta x$ with $\underline{\alpha} \in \mathbb{R}^{3}$ and $\beta \in \mathbb{R} ; \operatorname{div} \mathbf{q}_{2, h}=0$ implies that $\beta=0$ and $\mathbf{q}_{2, h}=\underline{\alpha}$.

It follows that

$$
\int_{T}\left(\mathbf{x}-\mathbf{x}_{T}\right) \mathbf{q}_{2, h} \mathrm{~d} x=\mathbf{q}_{2, h} \int_{T}\left(\mathbf{x}-\mathbf{x}_{T}\right) \mathrm{d} x=0
$$

so as $\operatorname{div} \mathbf{q}_{2, h}=0$ and $\left.q_{2, h}\right|_{T}=\underline{\alpha}$, forall $T \in \mathcal{T}_{2, h}$,

$$
\sum_{T \in \mathcal{T}_{2, h}} \int_{T} \operatorname{grad} u_{2, h} \mathbf{q}_{2, h} \mathrm{~d} x=\sum_{T \in \mathcal{T}_{2, h}} \int_{\partial T} u_{2, h} \mathbf{q}_{2, h} \cdot n_{T} \mathrm{~d} \sigma=\sum_{T \in \mathcal{T}_{2, h}} \sum_{e \text { edge of } \partial T} \overline{u_{2, h}} \mid e \int_{e} \mathbf{q}_{2, h} . n_{T} \mathrm{~d} \sigma
$$

where $\overline{u_{2, h} \mid e}=\int_{e} u_{2, h} \mathrm{~d} \sigma$. Since $\mathbf{q}_{2, h} \in H\left(\operatorname{div}, \Omega_{2}\right)$, we have

$$
\mathbf{q}_{2, h} \cdot n_{T_{1} \mid T_{1}}+\mathbf{q}_{2, h} \cdot n_{T_{2} \mid T_{2}}=0 \quad \text { on } e=\partial T_{1} \cap \partial T_{2},
$$

and $u_{2, h} \in V_{2, h}$ implies

$$
\overline{\left(u_{2, h \mid T_{1}}\right)_{\mid e}}=\overline{\left(u_{2, h \mid T_{2}}\right)_{\mid e}} .
$$

On the faces $e$ included in $\partial \Omega$, the contribution equals zero due to Drichlet condition. The non zero terms corresponding to faces included in $\Sigma$, we have finally

$$
\int_{\Omega_{2}} \mathbf{p}_{2, h} \cdot \mathbf{q}_{2, h} \mathrm{~d} x=\int_{\Sigma} u_{2, h} \mathbf{q}_{2, h} . n_{\Sigma} \mathrm{d} \sigma .
$$


As $\mathbf{q}_{2, h} . n_{T} \in \Lambda_{h}$ we get, from second equations of problem $\left(P_{H D}\right)$ :

$$
\int_{\Sigma} u_{2, h} \mathbf{q}_{2, h} \cdot n_{\Sigma} \mathrm{d} \sigma=\int_{\Sigma} u_{1, h} \mathbf{q}_{2, h} \cdot n_{\Sigma} \mathrm{d} \sigma
$$

and then the result

$$
\int_{\Omega_{2}} \mathbf{p}_{2, h} \mathbf{q}_{2, h} \mathrm{~d} x=\int_{\Sigma} u_{1, h} \mathbf{q}_{2, h} \cdot n_{\Sigma} \mathrm{d} \sigma
$$

Lemma 4.4. The function $\mathbf{p}_{2, h}$ satisfies:

$$
\sum_{T \in \mathcal{T}_{2, h}} \int_{T} \operatorname{grad} u_{2, h} \operatorname{grad} v_{2, h} \mathrm{~d} x=\int_{\Sigma} \mathbf{p}_{2, h} . n_{\Sigma} v_{2, h} \mathrm{~d} \sigma+\int_{\Omega_{2}} \bar{f} v_{2, h} \mathrm{~d} x, \forall v_{2, h} \in V_{2, h}
$$

Proof. For every $v_{2, h} \in V_{2, h}$, since $\operatorname{grad} v_{2, h} \in\left(P_{0}(T)\right)^{3}$ and $\int_{T}\left(\mathbf{x}-\mathbf{x}_{T}\right) \mathrm{d} x=0$, we have

$$
\begin{aligned}
\sum_{T \in \mathcal{T}_{2, h}} \int_{T} \operatorname{grad} u_{2, h} \operatorname{grad} v_{2, h} & =\sum_{T \in \mathcal{T}_{2, h}} \int_{T} \mathbf{p}_{2, h} \operatorname{grad} v_{2, h}+\sum_{T \in \mathcal{T}_{2, h}} \frac{\bar{f}}{3} \int_{T}\left(\mathbf{x}-\mathbf{x}_{T}\right) \mathrm{d} x \\
& =\sum_{T \in \mathcal{T}_{2, h}} \int_{T} \mathbf{p}_{2, h} \operatorname{grad} v_{2, h}-\sum_{T \in \mathcal{T}_{2, h}} \int_{T} \operatorname{div} \mathbf{p}_{2, h} v_{2, h} \mathrm{~d} x+\int_{\Sigma} \mathbf{p}_{2, h} . n_{\Sigma} v_{2, h} \mathrm{~d} \sigma \\
& =\int_{\Sigma} \mathbf{p}_{2, h} . n_{\Sigma} v_{2, h} \mathrm{~d} \sigma+\int_{\Omega_{2}} \bar{f} v_{2, h} \mathrm{~d} x .
\end{aligned}
$$

Lemma 4.5. The function $\mathbf{p}_{2, h}$ satisfies:

$$
\mathbf{p}_{2, h} \cdot n_{\Sigma}=-\lambda_{h} \text { on } \Sigma \text {. }
$$

Proof. Comparing the equation established in Lemma 4.7 with the discrete hybrid conforming-nonconforming formulation, and remarking that $\lambda_{h}$ is unique, and $\mathbf{p}_{2, h} . n_{\Sigma} \in \Lambda_{h}$, the result is deduced.

Theorem 4.1. The functions $\left(u_{1, h}, \mathbf{p}_{2, h}\right)$ are solutions of the following semi-primal semi-dual formulation $\left(P_{s}\right)$ :

$$
\left\{\begin{array}{l}
\text { Find }\left(u_{1, h}, \mathbf{p}_{2, h}\right) \in V_{1, h} \times W_{2, h} \text { such that } \\
\forall v_{1, h} \in V_{1, h}, \int_{\Omega_{1}} \operatorname{grad} u_{1, h} \operatorname{grad} v_{1, h} \mathrm{~d} x+\int_{\Sigma} \mathbf{p}_{2, h} . n_{\Sigma} v_{1, h} \mathrm{~d} \sigma=\int_{\Omega_{1}} f v_{1, h} \mathrm{~d} x, \\
-\operatorname{div} \mathbf{p}_{2, h}=\bar{f}, \quad \text { on } \Omega_{2}, \\
\forall \mathbf{q}_{2, h} \in W_{2, h}, \text { with } \operatorname{div} \mathbf{q}_{2, h}=0, \quad \int_{\Omega_{2}} \mathbf{p}_{2, h} \cdot \mathbf{q}_{2, h} \mathrm{~d} x-\int_{\Sigma} u_{1, h} \mathbf{q}_{2, h} . n_{\Sigma} \mathrm{d} \sigma=0 .
\end{array}\right.
$$

Theorem 4.2. If the solution $u$ to $\left(P_{H}\right)$ is such that $u_{i}=u_{\mid \Omega_{i}} \in H^{\sigma_{i}}\left(\Omega_{i}\right)$ with $1<\sigma_{i} \leq 2$, $i=1$,2, then we have the following error bound:

$$
\left|u-u_{1, h}\right|_{1, \Omega_{1}}+\left\|\mathbf{p}-\mathbf{p}_{2, h}\right\|_{0, \Omega_{2}} \leq C\left\{h_{1}^{\sigma_{1}-1}|u|_{\sigma_{1}, \Omega_{1}}+h_{2}^{\sigma_{2}-1}|u|_{\sigma_{2}, \Omega_{2}}+h_{2}|f|_{0, \Omega_{2}}\right\},
$$

where $\mathbf{p}=\operatorname{grad} u$.

Proof. From the $\mathbf{p}_{2, h}$ definition one gets:

$$
\left\|\mathbf{p}-\mathbf{p}_{2, h}\right\|_{0, T} \leq \sum_{T \in \mathcal{T}_{2, h}} \int_{T}\left|u-u_{2, h}\right|_{1, T}+\sum_{T \in \mathcal{T}_{2, h}}\left\|\frac{\bar{f}}{3}\left(x-x_{T}\right)\right\|_{0, T}, \text { where } \mathbf{p}=\operatorname{grad} u,
$$


and global error splits into three terms:

$$
\left|u-u_{1, h}\right|_{1, \Omega_{1}}+\left\|\mathbf{p}-\mathbf{p}_{2, h}\right\|_{0, \Omega_{2}} \leq\left|u-u_{1, h}-\right|_{1, \Omega_{1}}+\sum_{T \in \mathcal{T}_{2, h}} \int_{T}\left|u-u_{2, h}\right|_{1, T}+\sum_{T \in \mathcal{T}_{2, h}}\left\|\frac{\bar{f}}{3}\left(\mathbf{x}-\mathbf{x}_{T}\right)\right\|_{0, T} .
$$

The final result follows from the error bounds established in Theorem 4 and easy calculations.

\section{Conclusion}

Theorem 4.9. shows that $\left(u_{1, h}, \mathbf{p}_{2, h}\right)$ is solution of a semi-primal semi-dual formulation, therefore both of the unknowns are the approximation of the exact solution restriction to each subdomain. The continuity of $\mathbf{p}_{2, h}$ across tetrahedrons interfaces is obtained by local correction of grad $u_{2, h}$. This property is interesting, because numerically a nonconforming allows to obtain a more powerful approximation, verifying flux compatibility.

Theorem 4.10 gives approximation error bounds, which are optimal without any compatibility condition on the meshes. Moreover, one can note that the last term in the bound is dominated by the other two. In order to provide a very flexible method, regularity required on the exact solution is weak, and may not be the same on the two subdomains.

\section{REFERENCES}

[1] A. Agouzal, A Posteriori Error Estimator for Nonconforming Finite Element Methods. Appl. Math. Lett. 7 (1994) 61-66.

[2] A. Agouzal, Méthode de décomposition de domaines en formulation mixte. Jap. Math. 43 (1994) 31-35.

[3] A. Agouzal and J.-M.Thomas, Une méthode d'éléments finis hybrides en décomposition de domaines. RAIRO Modél. Math. Anal. Numér. 29 (1995) 749-764.

[4] A. Agouzal and L. Lamoulie, Un algorithme de résolution pour une méthode de décomposition de domaines par éléments finis. C.R. Acad. Sci. Paris Sér. I 318 (1994) 117-176.

[5] A. Alonso and A. Valli, A new approach to the coupling of viscous and inviscid Stokes equations. East-West J. Numer. Math. 3 (1995) 29-42.

[6] C. Bernardi, Y. Maday and A. Patera, A new nonconforming approach to domain decomposition: The mortar element method, in Nonlinear Partial Differential Equations and their Applications, H. Brezis and J.L. Lions Eds., Pitman (1989).

[7] F. Brezzi and M. Fortin, Mixed and Hybrid Finite Element Methods. Springer Verlag, New York (1991).

[8] P.G. Ciarlet, Basic Error Estimates for Elliptic Problems, in Handbook of Numerical Analysis, Vol II, Finite Element Methods, P.G. Ciarlet and J.L. Lions Eds., North-Holland, Amsterdam (1991) 17-352.

[9] P. Clement, Approximation by finite elements functions using local regularization. RAIRO Anal. Numér. 9 (1975) $77-84$.

[10] L. Lamoulie and J.-M.Thomas, Couplage de méthodes primales et duales d'éléments finis pour les problèmes elliptiques du second ordre. C.R. Acad. Sci. Paris Sér. I 318 (1994) 269-274.

[11] D. Marini, An inexpensive method for the evaluation of the solution of the lowest order Raviart-Thomas mixed method. SIAM J. Numer. Anal. 22 (1985) 493-496.

[12] J.E. Roberts and J.-M.Thomas, Mixed and Hybrid Methods, in Handbook of Numerical Analysis, Vol II, Finite Element Methods, P.G. Ciarlet and J.L. Lions Eds., North-Holland, Amsterdam (1991) 523-639.

[13] J.-M. Thomas, Finite Element Matching Methods, in Fifth International Symposium on Domain Decomposition Methods for Partial Differential Equations, D. Keys, T. Chan, G. Meurant, J. Scroggs and R. Voigt Eds., SIAM, Philadelphia (1992) 99-105. 